\title{
Theodor Kocher und Zürich
}

\author{
von Kurt Ritzmann
}

\section{ZUSAMMENFASSUNG}

Mannigfache Beziehungen verbanden Theodor Kocher mit Zürich. Hier verbrachte er sein letztes Hochschulsemester, wobei der Kontakt mit seinen Lehrern Friedrich Horner und Theodor Billroth für seine Wahl zum Professor in Bern nicht ohne Bedeutung war. Mit seinen Zürcher Kollegen Rudolf Ulrich Krönlein und Ferdinand Sauerbruch pflegte Kocher freundschaftlichen Umgang. Trotz vieler Gemeinsamkeiten stimmten die beiden, der gleichen Generation angehörenden Schweizer Kocher und Krönlein in ihrer Meinung nicht immer überein.

\section{Frühe Beziehungen}

Am 8. Mai 1865 bezog Theodor Kocher (1841-1917) im Oberhof bei der Platte Quartier ${ }^{1}$ und immatrikulierte sich an der Zürcher Hochschule ${ }^{2}$. Er belegte den chirurgischen Operationskurs von Theodor Billroth (18291894), den Sektionskurs des Pathologen Eduard Rindfleisch (1836-1908), die ophthalmologische Klinik und die Vorlesung «Die Augenheilkunde und die medizinische Klinik» bei Friedrich Horner (1831-1886), ferner die Kliniken von Anton Biermer (1827-1892) und Billroth ${ }^{3}$.

Kochers Doktor-Vater, Biermer, hatte im Frühjahr jenes Jahres Bern verlassen, um in Zürich das Ordinariat für innere Medizin zu übernehmen. Wir wissen aus Briefen Kochers, dass Biermer ihm versprochen hatte, die Ergebnisse der handschriftlich bereits vorliegenden Dissertation über «Behandlung der croupösen Pneumonie mit Veratrum-Präparaten» an weiteren Fällen zu überprüfen ${ }^{4}$. Daher bewarb sich Kocher für das Sommersemester um eine Unterassistentenstelle bei Biermer ${ }^{5}$.

Billroth gründete am 20. Mai 1865 ein «Medizinerkränzchen». Es sollte Professoren und Studierende zu lebhafterem Gedankenaustausch vereinigen. Nach dem Vortrag eines Studenten, der nicht länger als eine Stunde dauern durfte, folgte ein geselliger Teil. Billroth wurde zum ersten Präsiden- 
ten gewählt, und Biermer schlug «das Fieber» als ersten Gegenstand für Vorträge vor ${ }^{6}$.

Kocher nahm an diesem «Billroth-Kränzchen» regelmässig alle vierzehn Tage teil und beteiligte sich laut Protokoll oft an der Diskussion. Im Juni hielten Kocher und Adolf Broder (1838-1882) ${ }^{7}$ Vorträge «Über die neueren Fiebertheorien». Kocher verfocht die Ansicht, dass die Temperaturerhöhung wesentlich durch vermehrte Wärmebildung entstehe, dagegen Broder die Theorie, wonach sie von verminderter Wärmeabgabe abhänge ${ }^{8}$.

Als Frau Hirzel (1820-1903), die Gattin des Pfarrers zu Sankt Peter, wegen einer Eierstockgeschwulst in Behandlung zu Bernhard Breslau (1829-1866), dem Ordinarius für Frauenheilkunde, kam, nahm auf dessen Empfehlung Thomas Spencer Wells (1818-1897) im Juli den schwierigen Eingriff vor. Die Geschwulst wog über 50 Pfund ${ }^{9}$. Kocher wohnte der Operation in der alten Gebäranstalt ${ }^{10}$ bei. Ein Jahr später konnte er bei seinem Besuch in London Wells vom Wohlbefinden jener Patientin berichten. Wie er nach Hause schrieb, kam er durch diese günstigen Umstände Wells nahe ${ }^{11}$.

Im Herbst 1865 verliess Kocher Zürich «ohne Zeugnis», wie es im Matrikelbuch der Hochschule heisst ${ }^{2}$, da er sein Studium bereits vorher in Bern mit neun Semestern abgeschlossen hatte ${ }^{12}$.

Nachdem Kocher von der Berner Erziehungsdirektion 1866 die Venia docendi für Chirurgie erhalten hatte, bewarb er sich am 29. Februar 1872 um die Nachfolge Albert Lückes (1829-1894), der nach Strassburg ging. Von der Fakultät wurde Kocher an zweiter Stelle vorgeschlagen ${ }^{13}$.

Zur Wahl Kochers hat Zürich nicht unwesentlich beigetragen. Der Ophthalmologe Horner fragte Billroth, der nun in Wien war, beiläufig nach seiner Meinung über Kocher. Horner selber hatte ihn - wie er schrieb - «als tüchtigen Kopf, fleissigen Arbeiter und sehr umgänglichen, bescheidenen Menschen» in Erinnerung behalten ${ }^{13}$.

Bereits fünf Tage später erhielt Horner die ausführliche Antwort Billroths, Kocher sei «sehr talentvoll, ausserordentlich tüchtig in allen seinen Arbeiten, fleissig und sorgfältig in allem seinem Thun». Seine Arbeiten trügen den Stempel der Solidität und echter Wissenschaftlichkeit. Er sei vorzüglich zum Kliniker befähigt, so dass es diesmal nicht nötig sei, einen Ausländer hereinzurufen, da sich die Berner selbst eine so tüchtige Kraft herangezogen hätten. Horner leitete Billroths Brief an einen Berner Regierungsrat, dessen Schwägerin er behandelt hatte, weiter ${ }^{13}$.

Auch Bernhard von Langenbeck (1810-1887) in Berlin hatte sich für 
Kocher eingesetzt. Die Schreiben Billroths und Langenbecks bestimmten die Behörden, den Eingaben der Studierenden und der Berner Ärzte zu entsprechen ${ }^{13}$.

Mit 31 Jahren wurde Kocher Professor der Chirurgie. Zwölf Jahre später schrieb er in einem Brief an seinen ehemaligen Assistenten, den Anatomen Hans Strasser (1852-1927):

«Ich weiss von mir selber, dass das frühe Drankommen an die Professur einen im Verlauf noch recht viel Sorge und Mühe bereitet und dass alle Arbeit vorher doppelt leicht ist.» ${ }^{14}$

45 Jahre dauerte die Ära Kocher in Bern. Wie August Socin (1837-1899) in Basel und Rudolf Ulrich Krönlein (1847-1910) in Zürich blieb Kocher trotz Berufungen ins Ausland seinem angestammten Wirkungskreis treu. In Zürich wirkten in der gleichen Zeitspanne drei Professoren.

\section{Kocher und seine Zürcher Kollegen}

Edmund Rose (1836-1914) war 1867 als Nachfolger Billroths aus Berlin berufen worden. Er war ebenfalls Kropfchirurg; 1877 sprach er am Deutschen Chirurgen-Kongress in Berlin «Über Totalexstirpation der Strumen». ${ }^{15}$

1880 wollte sich der ehemalige Kocher-Assistent Constantin Kaufmann (1853-1934) in Zürich habilitieren. Rose hielt seine Arbeiten für «nicht gründlich genug» ${ }^{16}$. Die Fakultät erkundigte sich hierauf bei Kocher und erhielt die Antwort, dass es nicht recht verständlich sei, weshalb man in Zürich so viele Umstände mache. Kaufmann sei ein Mann von aussergewöhnlicher Arbeitskraft, ganz gediegenen Kenntnissen; er habe einen sehr guten Vortrag und könne der Fakultät nur zur Zierde gereichen ${ }^{17}$.

Als Rose 1881 Zürich verliess, wurde Krönlein sein Nachfolger. Er hatte sich zehn Jahre bei Rose und Langenbeck ausgebildet und war in Berlin ausserordentlicher Professor geworden. Trotzdem schlug die Fakultät ihn erst an vierter Stelle zur Wahl vor ${ }^{18}$. Wie bei Kocher setzten sich Langenbeck und Billroth für den 33jährigen Schweizer ein. Billroth nannte in einem Schreiben auch Kocher, meinte jedoch, er würde gewiss nicht nach Zürich kommen, da er in Bern eine gute Position habe und durch den Wechsel kaum etwas gewänne ${ }^{19}$.

Krönleins Wahl beurteilte Billroth in einem Brief an Horner mit den Worten: «Ich meine, die Schweizer müssten sehr stolz sein, dass sie aus eigenen Kräften jetzt drei Schweizer als Kliniker in Zürich haben ${ }^{20}$; das war 
noch nicht da. Sie, Krönlein, Kocher, Socin sind denn doch Leute, auf deren Besitz jede deutsche Universität stolz sein würde.» ${ }^{21}$

Es gibt im Leben von Kocher und Krönlein viele Gemeinsamkeiten. Beide entstammten dem Mittelstand, beide hatten fünf Brüder und eine Schwester, beide waren als Gymnasiasten stets Primus ihrer Klasse und hatten ein Zeichentalent ${ }^{22}$.

Wie Kocher in Bern hat Krönlein in seinen dreissig Zürcher Jahren entscheidend zum Aufschwung der Chirurgie beigetragen. Beide waren vielseitig, sie operierten ruhig, sicher und schonend. Als Lehrer trugen beide eindringlich und klar, nüchtern und ernst vor. Nicht nur chirurgisches Wissen und Können, sondern auch humanes Denken und Fühlen übertrugen sie auf ihre Schüler. Gewissenhaftigkeit verlangten beide von ihren Assistenten. Doch während Kocher sie bis zur Erschöpfung belastete, liess Krönlein ihnen viel Freiheit. Menschlich erschienen beide zurückhaltend ${ }^{23}$.

Beide waren Präsidenten der lokalen Ärztegesellschaften und stellten sich auch dem Centralverein als Mitglieder des ständigen Ausschusses zur Verfügung ${ }^{24}$.

So parallel auch die berufliche Laufbahn von Kocher und Krönlein verlief - einer Meinung waren sie längst nicht immer. Nachdem beide zu Vorsitzenden der Deutschen Gesellschaft für Chirurgie gewählt worden waren, stieg Kocher die Stufenleiter der Ehrungen empor und wurde 1905 zum Vorsitzenden des ersten internationalen Chirurgen-Kongresses in Brüssel ernannt. Krönlein hielt diese Veranstaltung für ganz unnötig und hoffte auf eine Zeit, wo der internationale Kongressschwindel aufhöre, wodurch die Wissenschaft nicht verlöre, wohl eher gewänne ${ }^{25}$.

Auch mit der Zusammensetzung der Delegationen war Krönlein nicht einverstanden. Es handle sich dabei um die «Mache einiger weniger strebender Leute»; anfänglich waren es fast ausschliesslich Berner Ärzte ${ }^{25}$. Bereits 1890 hatte der Augenarzt Otto Haab (1850-1931) in der Zürcher Fakultät die selbstherrliche Bestimmung einer Schweizer Delegation durch Kocher kritisiert ${ }^{26}$.

Als Kocher in einer Arbeit «volle Sicherheit» und «Garantie» für seine Nahttechnik am Magen versprach ${ }^{27}$, erhob Krönlein 1896 den Einwand, dass das Wort «Garantie» sich im Munde des Arztes immer etwas bedenklich ausnehme. Es bringe ihn in Gefahr, mehr zu versprechen, als er halten könne. Die ausgezeichneten Resultate Kochers seien mehr seiner hervorragenden operativen Technik überhaupt als gerade seiner speziellen Methode zuzuschreiben ${ }^{28}$. 
Es spricht für Kocher, dass er das Bedürfnis hatte, die Wogen zu glätten. Aus den Briefen an seine Frau geht hervor, dass er in Zürich Krönlein aufsuchte, um mit ihm ein freundschaftliches Wort zu reden, das breche doch mancher Bitterkeit die Spitze. Tatsächlich verlief die Begegnung erfreulich und stellte die alten Beziehungen wieder her ${ }^{29}$.

Der Ausgleich durch die eigene Familie fehlte bei Krönlein, so dass die seelischen Aufregungen, die der Beruf mit sich brachte, zunehmend schwer auf ihm lasteten. Kocher fand sowohl in seiner Familie als auch in seinem unerschütterlichen Gottvertrauen festen Halt ${ }^{30}$. Er war Christ, Krönlein dagegen Humanist.

Nach Krönleins Rücktritt wurde auch Kocher wegen Kandidaten für die Nachfolge angefragt. Er empfahl seinen Schüler Ernst Tavel (1858-1912), den die Kommission jedoch nicht auf die Liste nahm, da er ein schlechter Lehrer sei ${ }^{31}$.

Als Conrad Brunner (1859-1927) in Münsterlingen den Ruf nach Zürich abgelehnt hatte, schrieb ihm Kocher, er hätte sich sehr gefreut, ihn als Kollegen zu begrüssen und öfters mit ihm zusammenzutreffen.

«Allein ich achte Ihren Standpunkt in höchstem Masse und wünsche mir solche treue Assistenten, wie Krönlein in Ihnen einen hat, die es nicht dulden, dass ihr Lehrer verunglimpft wird.» ${ }^{32}$

Hierauf wurde der 35jährige Ferdinand Sauerbruch (1875-1951) aus Marburg gewählt. Bald fanden sich die Lungenspezialisten aus den Schweizer Tuberkulosezentren bei ihm ein, um bei Operationen zuzusehen. Auch Kocher kam. Er quartierte sich mit zwei Söhnen ein paar Wochen lang in der Stadt ein, sofern wir den Angaben Sauerbruchs Glauben schenken dürfen. Sie sollen sich jede Operation angesehen haben ${ }^{33}$.

Bei seinen Besuchen in deutschen Kriegslazaretten fuhr Kocher noch 1917 nach Singen und liess sich von Sauerbruch seine Handprothesen vorführen ${ }^{34}$.

Bei der Abdankung für Kocher in der Heiliggeistkirche in Bern sprach Sauerbruch als Vertreter der Deutschen Gesellschaft für Chirurgie. Zu Beginn des folgenden Wintersemesters hielt er vor den Klinikern die Gedächtnisrede ${ }^{35}$. Die Worte Sauerbruchs gehören zum Besten, was über Kocher gesprochen worden ist.

Ausser zu den Chirurgieprofessoren bestanden zwischen Kocher und Zürich noch weitere Bande. Der Zürcher Neurologe Constantin von Monakow (1853-1930) berichtet, dass Kocher sich bei ihm Präparate angesehen 
und zur Mitarbeit an seinem «Archiv für Psychiatrie und Neurologie» bereit gefunden habe ${ }^{36}$.

Ein enger Freund Kochers, Walter Krebs (1847-1925), vormals Arzt in Herzogenbuchsee, verbrachte seine letzten Lebensjahre bei seiner Tochter Maria Waser (1878-1939) in Zollikon bei Zürich ${ }^{37}$.

Zwei Kocher-Assistenten sind Zürcher Ordinarien geworden: Max Walthard (1867-1933) 1920 für Frauenheilkunde ${ }^{38}$, und Ernst Hedinger (18731924) 1922 für Pathologie ${ }^{39}$.

Der Kocher-Schüler und Chirurgieprofessor in Bern, Carl Arnd (18651923), schrieb 1913 bei der Gründung der Schweizer Gesellschaft für Chirurgie, dass man eine solche Vereinigung schon vor Jahren geplant habe. Wörtlich heisst es:

«Wir gaben den Versuch sofort auf, weil wir in der Schweiz zwei zu grosse Chirurgen hatten, um einig werden zu können. Für beide Fürsten schien kein Platz vorhanden! An die Fürsten traten wir gar nicht einmal heran, die Minister genügten uns schon, um uns Angst zu machen.» ${ }^{40}$

\section{Anmerkungen}

1 Stadtarchiv Zürich VI Fl C 53, Verzeichnis der in der Gemeinde Fluntern wohnenden Studierenden.

2 Staatsarchiv Zürich UU 24 a, Matrikelbuch der Hochschule.

3 Staatsarchiv Zürich UU 25, Honorargebühren der Studenten.

4 Bonjour, Edgar, Theodor Kocher, Bern 1981, S. $19 \mathrm{f}$.

5 Staatsarchiv Zürich U 107 b (fasc. 2 b), Assistenten und Unterassistenten von Biermer.

6 Universitätsspital-Bibliothek Zürich, Protokollbuch des Medizinerkränzchens, S. 1.

7 Später Arzt in Sargans im «Broderhaus».

8 Wie 6, S.2.

Kocher war bereits im schriftlichen Doktorexamen am 24. März 1865 von Biermer über «Fieber» geprüft worden.

9 Correspondenz-Blatt für Schweizer Ärzte 34 (1904), S. 70 f.

10 Corresp.-Bl.f. Schweiz. Ärzte 24 (1894), S. 163 (Kappeler, Otto, Zur Erinnerung an Theodor Billroth).

11 Bonjour (s. Anm.4), S.25.

12 Medizinhistorisches Institut Bern, Protokolle I der med. Fakultät, S. 308.

13 Staatsarchiv Bern BB III b 555, Personaldossier Kocher.

14. Medizinhistorisches Institut Zürich, Brief von Theodor Kocher an Hans Strasser, undatiert (auf Grund des Inhaltes Datierung September 1884 möglich). 
15 Verhandlungen der Deutschen Gesellschaft für Chirurgie 1877, S.114. Der Vortrag von Edmund Rose ist auf S. 75-145 in erweiterter Form abgedruckt unter dem Titel: Über den Kropftod und die Radicalcur der Kröpfe.

16 Universitätsarchiv Zürich, Protocoll der Medicinischen Fakultät Zürich, S. 112.

17 Medizinhistorisches Institut Zürich, Brief von Theodor Kocher an August Forel, Aktuar der Fakultät, 9. Mai 1880.

18 Wie 16, S.116, und Staatsarchiv Zürich UU 2.31.

19 Medizinhistorisches Institut Zürich, Brief von Theodor Billroth an Friedrich Horner, 11. November 1880.

20 Horner, Oscar Wyss (1840-1918) und Krönlein.

21 Medizinhistorisches Institut Zürich, Brief von Theodor Billroth an Friedrich Horner, 16. Januar 1881.

22 Für Kocher: Bonjour (s. Anm.4), S.12f.; Arnd, Carl, Theodor Kocher, Schriften des bernischen Hochschulvereins, Nr. 3, Bern 1917, S.2.

Für Krönlein: Familienschein. Programm der Prüfungen am Gymnasium Schaffhausen, Schaffhausen 1863-1866. Zeichnungen im Familienbesitz.

Bei der Habilitation von Krönlein in Berlin hob der Dekan Adolf Bardeleben im Gutachten über die eingereichten Arbeiten vom 30. November 1874 die «sehr guten, vom Verfasser selbst gezeichneten Abbildungen» hervor.

23 Für Kocher: Bonjour, S. 63 f., 70 f., 79; Garrè, Carl, Th. K. In: Deutsche med. Wochenschrift 43 (1917), S. 1112; Quervain, Fritz de, Erinnerungen aus der Kocherschen Klinik. In: Der Chirurg 2 (1930), S.1133 f.; Gröbly, Walter, Erinnerungen an Th. K. In: Schweiz. med. Wochenschr. 71 (1941), S.1028, $1030 \mathrm{f}$.

Für Krönlein: Wettstein, Albert, R. U.K. In: Schweiz. Rundschau f.Med. 1910, S.1416; Brunner, Conrad, R. U. K. In: Corresp.-Bl.f. Schweiz. Arzte 41 (1911), S. 12 f.

24 Hintzsche, Erich, Medizin und Mediziner seit 1870, Basel 1971, S. 78, 106, 142. Kocher war 1885 bis 1899, Krönlein 1890 bis 1899 Mitglied des ständigen Ausschusses.

25 Nachlass Conrad Brunner (Familienbesitz), Brief von Rudolf Ulrich Krönlein an Conrad Brunner, 23. Februar 1907.

26 Universitätsarchiv Zürich, Protocoll der Medicinischen Fakultät Zürich, S. 7.

27 Kocher, Theodor, Methode und Erfolge der Magenresection wegen Carcinom. In: Deutsche med. Wochenschr. 21 (1895), S. 289 f.

28 Krönlein, Rudolf Ulrich, Chirurgische Erfahrungen über das Magencarcinom. In: Beiträge zur klinischen Chirurgie 15 (1896), S. 334.

29 Bonjour (s. Anm.4), S.49, und briefliche Mitteilung von Prof. Edgar Bonjour, 19. Januar 1991.

30 Bonjour, S.87, 92, 95 f.; Gröbly, op. cit. (in Anm. 23).

31 Wie Anm. 26, S. 343.

32 Nachlass Conrad Brunner (Familienbesitz), Brief von Theodor Kocher an Conrad Brunner, undatiert.

33 Sauerbruch, Ferdinand, Das war mein Leben, Bad Wörishofen 1951, S. $174 \mathrm{f}$.

34 Ibidem, S. 305.

35 Sauerbruch, Ferdinand, Theodor Kocher. Gedächtnisrede zu Beginn der klinischen Vorlesungen in Zürich im Winter 1917. In: Münchener med. Wochenschr. 65 (1918), S. 78-80.

36 Monakow, Constantin von, Vita mea - mein Leben, Bern 1970, hg. v. A. W. Gubser und E. H. Ackerknecht, S. $268 \mathrm{f}$. 
37 Küffer, Georg, Maria Waser, Bern 1971, S.16.

38 Anderes, Ernst, Geschichte der kantonalen Frauenklinik. In: Zürcher Spitalgeschichte, Bd.2, Zürich 1951, S.296, und briefliche Mitteilung von Prof.Edgar Bonjour, 19. März 1991.

39 Briefliche Mitteilung von Prof. Christoph Hedinger, 8. April 1991, und Staatsarchiv Bern BB III b 2410.5.

40 Nachlass Conrad Brunner (Familienbesitz), Brief von Carl Arnd an Conrad Brunner, 13. Januar 1913.

\section{Summary}

\section{Theodor Kocher and Zurich}

Theodor Kocher was linked to Zurich by many relationships. It was in this town that he spent his last semester at university, and his contacts with his teachers Friedrich Horner and Theodor Billroth were of some importance with regard to his election as a professor at Berne. Kocher was on friendly terms with his Zurich colleagues Rudolf Ulrich Krönlein and Ferdinand Sauerbruch. In spite of many common views, however, the two Swiss surgeons Kocher and Krönlein were not always of the same opinion.

\section{Résumé}

\section{Theodor Kocher et Zurich}

Une quantité de relations liaient Theodor Kocher avec Zurich. C'est ici qu'il passait son dernier semestre d'université, et le contact avec ses professeurs Friedrich Horner et Theodor Billroth était certainement d'importance pour sa nomination comme professeur à Berne. Kocher se trouvait en termes amicaux avec ses collègues de Zurich, Rudolf Ulrich Krönlein et Ferdinand Sauerbruch. Malgré beaucoup de traits communs les deux Suisses Kocher et Krönlein n'étaient pas toujours de la même opinion.

Dr.med. Kurt Ritzmann

Schwalbenbodenstrasse 5 a

8832 Wollerau SZ 